University of Nebraska - Lincoln

DigitalCommons@University of Nebraska - Lincoln

3-22-2007

\title{
Nanoscale polarization manipulation and imaging of ferroelectric Langmuir-Blodgett polymer films
}

\author{
Brian J. Rodriguez \\ Oak Ridge National Laboratory, Oak Ridge, Tennessee \\ Stephen Jesse \\ Oak Ridge National Laboratory, sjesse@ornl.gov \\ Sergei V. Kalinin \\ Oak Ridge National Laboratory, Oak Ridge, Tennessee, sergei2@ornl.gov \\ Jihee Kim \\ University of Nebraska - Lincoln, jhkim@unlserve.unl.edu \\ Stephen Ducharme \\ University of Nebraska, sducharme1@unl.edu \\ See next page for additional authors
}

Follow this and additional works at: https://digitalcommons.unl.edu/physicsducharme

Part of the Physics Commons

Rodriguez, Brian J.; Jesse, Stephen; Kalinin, Sergei V.; Kim, Jihee; Ducharme, Stephen; and Fridkin, V.M., "Nanoscale polarization manipulation and imaging of ferroelectric Langmuir-Blodgett polymer films" (2007). Stephen Ducharme Publications. 29.

https://digitalcommons.unl.edu/physicsducharme/29

This Article is brought to you for free and open access by the Research Papers in Physics and Astronomy at DigitalCommons@University of Nebraska - Lincoln. It has been accepted for inclusion in Stephen Ducharme Publications by an authorized administrator of DigitalCommons@University of Nebraska - Lincoln. 


\section{Authors}

Brian J. Rodriguez, Stephen Jesse, Sergei V. Kalinin, Jihee Kim, Stephen Ducharme, and V.M. Fridkin 


\title{
Nanoscale polarization manipulation and imaging of ferroelectric Langmuir-Blodgett polymer films
}

\author{
Brian J. Rodriguez, ${ }^{\text {a) }}$ Stephen Jesse, and Sergei V. Kalinin ${ }^{\text {b) }}$ \\ Materials Science and Technology Division, Oak Ridge National Laboratory, Oak Ridge, Tennessee 37831 \\ Jihee Kim and Stephen Ducharme ${ }^{\mathrm{c})}$ \\ Department of Physics and Astronomy, University of Nebraska, Lincoln, Nebraska 68588-0111 and \\ Nebraska Center for Materials and Nanostructures, University of Nebraska, Lincoln, Nebraska 68588-0111 \\ V. M. Fridkin \\ Department of Physics and Astronomy, University of Nebraska, Lincoln, Nebraska 68588-0111; \\ Nebraska Center for Materials and Nanostructures, University of Nebraska, Lincoln, Nebraska 68588-0111; \\ and Institute of Crystallography, Russian Academy of Sciences, Moscow 117333, Russia
}

(Received 7 December 2006; accepted 15 February 2007; published online 22 March 2007)

\begin{abstract}
Piezoresponse force microscopy has been used to manipulate and image polarization of ferroelectric polymer Langmuir-Blodgett films at the nanoscale, achieving polarization control with a resolution below $50 \mathrm{~nm}$ and imaging resolution below $5 \mathrm{~nm}$. Individual regions showed square polarization-voltage hysteresis loops, demonstrating bistability of the polarization state. Arbitrary polarization patterns could be repeatedly written and erased with a resolution of 25 to $50 \mathrm{~nm}$, limited by grain size, demonstrating the potential for high-density data storage and retrieval at densities exceeding $250 \mathrm{Gbits} / \mathrm{in}^{2}$. (C) 2007 American Institute of Physics.
\end{abstract}

[DOI: $10.1063 / 1.2715102]$

The behavior of ferroelectricity at the nanoscale is the focus of increasing research activity because of intense interest in the fundamental nature of spontaneous order in condensed-matter systems and because of the many practical applications of ferroelectric thin films to electromechanical transducers, ${ }^{1}$ infrared imaging sensors, ${ }^{2}$ and nonvolatile memories. ${ }^{3}$ The ferroelectric polymer, polyvinylidene fluoride (PVDF), is being developed for use in nonvolatile data storage, ${ }^{4}$ and flexible ferroelectric electronic components due to its outstanding electromechanical, dielectric, and mechanical properties. ${ }^{5-7}$ High-quality thin films of PVDF and its copolymers can be fabricated using a Langmuir-Blodgett (LB) technique. These two-dimensional ferroelectric films have allowed the intrinsic coercive field to be measured. ${ }^{8}$ In order to realize the full potential of PVDF for applications such as ultrahigh-density nonvolatile memories, significant progress must be made in nanoscale characterization of the structure and ferroelectric properties of PVDF LB films, including local hysteresis and switching dynamics. PVDF has been the focus of numerous scanning probe microscopy investigations, including the pioneering use of scanning near field acoustic microscopy, ${ }^{9}$ scanning tunneling microscopy (STM), ${ }^{10}$ and atomic force microscopy ${ }^{11}$ (AFM) for electromechanical imaging and polarization switching. Several recent piezoresponse force microscopy (PFM) studies of ferroelectric polymers ${ }^{12,13}$ and oligomers ${ }^{14,15}$ have been reported. Ferroelectric domains as small as $30 \mathrm{~nm}$ were created, ${ }^{12,13}$ and asymmetric piezoelectric behavior was reported in spincoated copolymer films of polyvinylidine fluoride and trifluroroethylene $\mathrm{P}(\mathrm{VDF}-\mathrm{TrFE})$. Atomic-resolution imaging of $\mathrm{P}(\mathrm{VDF}-\mathrm{TrFE})$ monolayers by has produced images of dis-

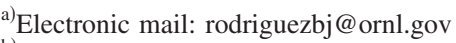

${ }^{\text {b) }}$ Author to whom correspondence should be addressed; electronic mail: sergei2@ornl.gov

${ }^{c}$ Electronic mail: sducharme@unl.edu
}

continuities in molecular conformation, which have been interpreted as domain walls perpendicular to the chain. ${ }^{16} \mathrm{PFM}$ has also been used to image polarization structure and local switching in $\mathrm{P}(\mathrm{VDF}-\mathrm{TrFE}) \mathrm{LB}$ films ${ }^{17}$ and nanotubes. ${ }^{18}$ Electrostatic force microscopy has been used to investigate the ferroelectric properties of LB P(VDF-TrFE) thin films; however, no domain structure was observed within crystals. ${ }^{19}$ Here, we report on nanoscale polarization imaging and polarization switching in LB films of $\mathrm{P}(\mathrm{VDF}-\mathrm{TrFE})$ by PFM, showing imaging resolution of $5 \mathrm{~nm}$ and switchable polarization in regions averaging $25-50 \mathrm{~nm}$ in size.

The thin ferroelectric $\mathrm{P}(\mathrm{VDF}-\mathrm{TrFE})$ copolymer films were fabricated on highly ordered pyrolytic graphite (HOPG) substrates by a LB technique. ${ }^{20}$ De-ionized water with a resistivity of $18 \mathrm{M} \Omega$ was used as a pure water subphase and placed in a clean Teflon trough with a total area of $1520 \mathrm{~cm}^{2}$. Then, a solution of vinylidene fluoride (70\%) with trifluoroethylene (30\%) copolymer, P(VDF-TrFE 70:30), in dimethyl sulfoxide ( $0.01 \%$ weight concentration) was dispersed on the top of the water with the aid of an electric pipette and microslides. The dispersed copolymer solution became a thin film on the water subphase, and the film was compressed at a rate of $20-60 \mathrm{~cm}^{2} / \mathrm{min}$ by two barriers from the outside towards the center of the trough to reach a target surface pressure of $5 \mathrm{mN} / \mathrm{m}$. Once the target pressure was reached, the film on the water surface was transferred to the HOPG substrate by horizontal dipping. The water trough was kept at $25^{\circ} \mathrm{C}$ during the entire process. Samples were then annealed for $1 \mathrm{~h}$ at $130{ }^{\circ} \mathrm{C}$ to optimize their crystal structure and stabilize the ferroelectric properties. ${ }^{21}$ Prior studies showed that this technique produces films with an average thickness of $1.78 \pm 0.07 \mathrm{~nm} / \mathrm{ML}$ (monolayer) transferred. ${ }^{22}$

Piezoresponse force microscopy studies were implemented on a commercial AFM system (Veeco MultiMode NS-IIIA) equipped with an external signal generation and data acquisition system. ${ }^{23}$ Measurements were performed us- 

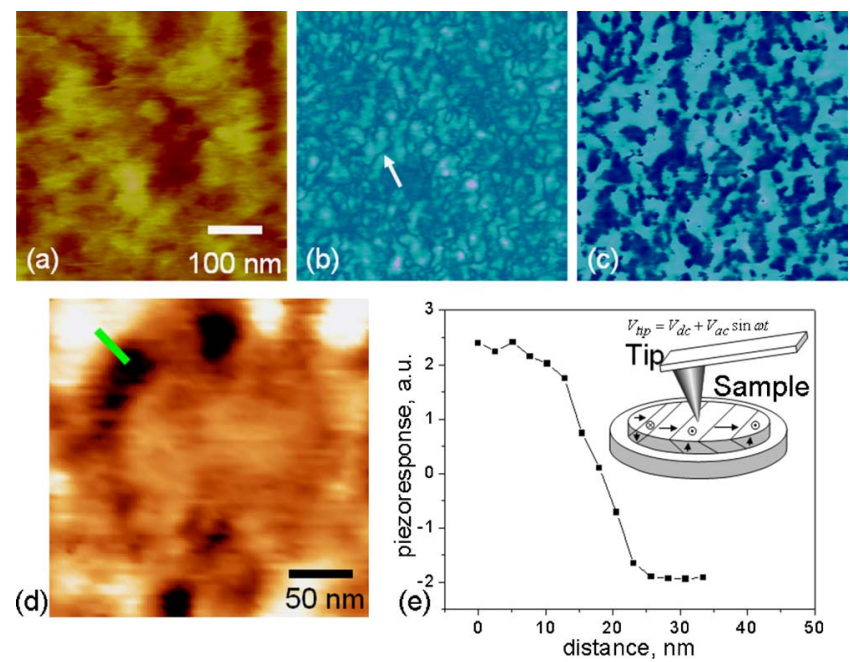

FIG. 1. (Color online) PFM data from a 10 ML LB film of P(VDF-TrFE 70:30) copolymer: (a) topography, (b) PFM amplitude, (c) PFM phase, (d) composite [amplitude $\times \cos$ (phase)] PFM, and (e) line scan of the composite image along the green bar.

ing $\mathrm{Au}-\mathrm{Cr}$ coated $\mathrm{Si}$ tips (Micromasch, spring constant $k$ $\sim 0.08 \mathrm{~N} / \mathrm{m}$ ). The images were acquired using a modulation voltage of $5 \mathrm{~V}_{\text {p.p. }}$ (electric field $\sim 250 \mathrm{MV} / \mathrm{m}$ ) at a frequency $(120 \mathrm{kHz})$ well above the first contact resonance.

Topography and PFM amplitude $\left(d_{1 \omega}\right)$ and phase $(\varphi)$ images are shown in Figs. 1(a)-1(c), respectively). The topography image [Fig. 1(a)] shows that the LB film is highly planar, with a root-mean-square roughness of $1.4 \pm 0.2 \mathrm{~nm}$. The PFM amplitude image [Fig. 1(b)] shows irregularly shaped regions of uniform piezoresponse, outlined by narrow unpolarized regions that appear to be intergrain boundaries, although this is less evident in the topography image [Fig. 1 (a) $]$ due to the loading $(\sim 0.4 \mathrm{nN})$ and capillary $(\sim 30-40 \mathrm{nN})$ forces associated with contact mode imaging. In the PFM phase image [Fig 1(c)], a bright region corresponds to a domain that has a polarization oriented toward the substrate, while a dark region corresponds to a region with polarization oriented away from the substrate, a "down" polarization and an "up" polarization, respectively. Boundaries can be seen in the PFM amplitude images [Fig. 1(b)] between two opposite polarizations [one such domain wall is indicated with an arrow in Fig. 1(b)]. This minimum in the amplitude signal corresponds to an apparently unpolarized boundary wall separating two grains. There is a distribution of topographic feature sizes between $\sim 25$ and $50 \mathrm{~nm}$ in diameter. For a polarization oriented perpendicular to the substrate, a field applied parallel to the dipole will result in a piezoresponse perpendicular to the substrate. The in-plane component of polarization was also measured (not shown), and the regions of uniform in-plane orientation correspond to the regions of uniform out-of-plane polarization.

To illustrate an imaging resolution, we generated a composite image [Fig. 1(d)] of the mixed piezoresponse, PR $=d_{1 \omega} \cos \varphi$, from the amplitude and phase images [Figs. 1(b) and 1(c)]. The composite image clearly shows domains of opposing polarization as bright up polarization and dark down polarization. A line profile across one of these grain boundaries is shown in Fig. 1(e). The width of the transition from positive to negative piezoresponse determines the imaging resolution, approximately $5 \mathrm{~nm}$ in this case, an order

of magnitude better than is typically observed for inorganic $5 \mathrm{~nm}$.
Downloaded 30 Mar 2007 to 129.93 .16 .206 . Redistribution subject to AlP license or copyright, see http://apl.aip.org/apl/copyright.jsp

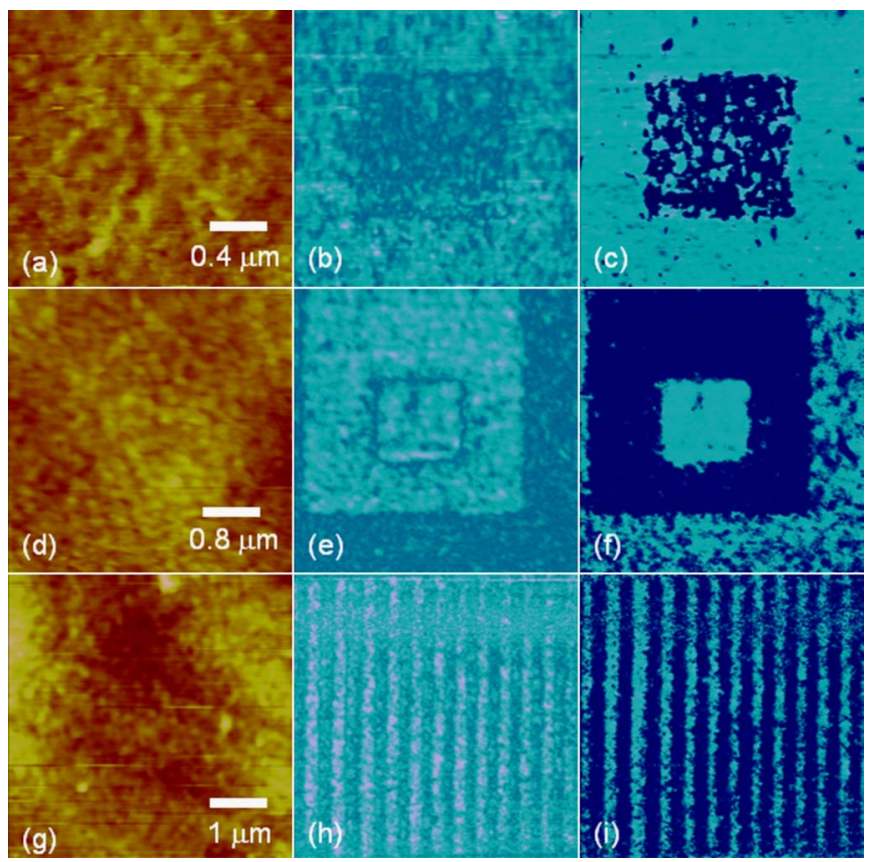

FIG. 2. (Color online) PFM images of patterns that were written with a tip bias of $\pm 10 \mathrm{~V}$ in a $10 \mathrm{ML}$ LB film of P(VDF-TrFE 70:30) copolymer: [(a), (d), and (g)] topography, [(b), (e), and (h)] PFM amplitude, and [(c), (f), and (i)] PFM phase. Patterns were written with $\pm 10 \mathrm{~V}$. The quality of the switched pattern depends on the voltage, the film, and the tip state. The domain walls are irregular and correspond to topographic features.

ferroelectrics. ${ }^{12,13}$ The high resolution is achieved because the LB films are highly planar, without complex lamellar morphologies that dominate solvent-formed films.

To investigate the polarization switching of LB P(VDFTrFE) films, a dc bias was applied while scanning in order to switch the polarization from its as-annealed state. First, a square area was poled in one direction by the application of a dc bias while scanning 256 lines at 1 line/s. Then, a smaller square area was poled in the opposite direction by the application of a dc bias of opposite polarity, and the entire region was imaged with an ac bias. In Figs. 2(a)-2(c) topography, PFM amplitude, and PFM phase images, respectively, are shown following poling with $\pm 10 \mathrm{~V}$ dc bias. In Figs. 2(d)-2(f), a larger area has been poled and imaged with the same conditions. As can be seen in the PFM images in Figs. 2(b), 2(c), 2(e), and 2(f), the regions of uniform polarization are rough and irregular, and correspond well to the topographic structure, suggesting that switching occurs one grain at a time, rather than on a multiple grain basis. The less uniform pattern in Figs. 2(b) and 2(c) is indicative of a degraded tip. Domains written using a square wave bias $( \pm 10 \mathrm{~V})$ pattern along the scanning direction produced the series of parallel lines shown in Figs. 2(g)-2(i). In general, domains were stable over the course of several hours, and could be rewritten several times over the course of a day . Interestingly, even though dipole rotation and conformation changes are inseparable given the covalent bonding of the chains, it is still possible to switch a single grain without rotating the polarization of nearby grains when the field is applied locally using a conductive AFM tip. The polarization direction of the grains of annealed P(VDF-TrFE) LB films can be determined and switched with a writing resolution corresponding to the grain size and an imaging resolution of $5 \mathrm{~nm}$.

to AIP license or copyright, see http://apl.aip.org/apl/copyright.jsp 

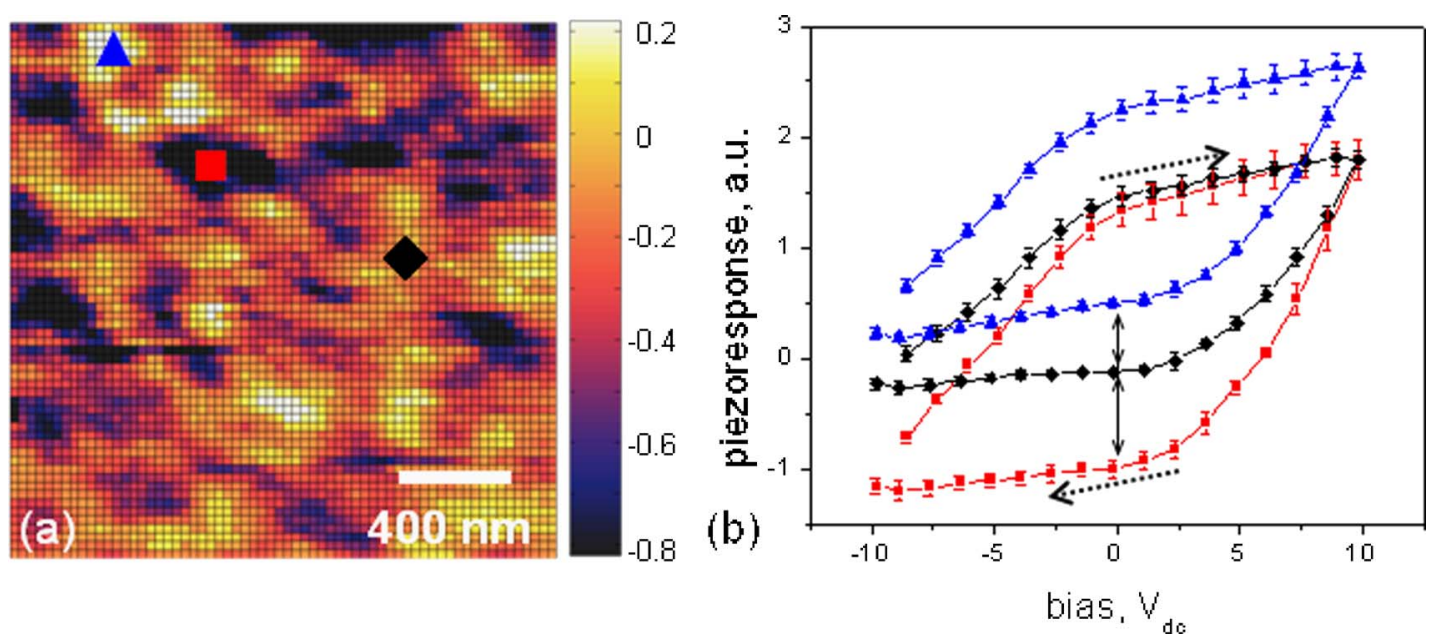

FIG. 3. (Color online) Polarization hysteresis loops obtained from a 10 ML Langmuir-Blodgett film of P(VDF-TrFE 70:30) copolymer: (a) SS-PFM image of the negative remanent piezoresponse for a $2 \times 2 \mu \mathrm{m}^{2}$ region (32 nm step size) and (b) representative hysteresis loops from regions indicated in (a). Sweep direction is indicated by dashed arrows.

To investigate the local switching properties in more detail, switching spectroscopy PFM (SS-PFM) was employed, in which a local hysteresis loop was measured at every point within a specified grid. ${ }^{23}$ The switching parameters (imprint, remanent piezoresponse, etc.) can then be extracted from the loops and plotted as two-dimensional maps. In this manner, the dynamics of the local switching characteristics can be probed and the switching properties can be visualized to show how these characteristics vary across a sample surface. ${ }^{23}$ In Fig. 3 a SS-PFM image of the negative remanent piezoresponse within a $2 \times 2 \mu \mathrm{m}^{2}$ area and representative hysteresis loops demonstrating a variation in the vertical shift are shown. The loops were measured with a driving voltage of $5 \mathrm{~V}_{\text {p.p. }}$ at a frequency of $710 \mathrm{kHz}$. Each loop is an average of two $\sim 30 \mathrm{~ms}$ wave form cycles. The hysteresis loops measured on $\mathrm{P}(\mathrm{VDF}-\mathrm{TrFE})$ do not saturate with the range of $\pm 10 \mathrm{~V}$ and attempt to bring the loops to saturation by increasing the dc bias typically ended with dielectric breakdown at biases of $10 \mathrm{~V}$ and higher depending on the location, precluding a systematic study of the switching properties. Slower cycling did not result in more saturated loops. The coercive field of similar copolymer films determined from pyroelectric hysteresis loops is $\sim 80 \mathrm{MV} / \mathrm{cm}^{24}$ $\sim 170 \mathrm{MV} / \mathrm{cm}$ lower than reported here, presumably due to the geometrical differences in the measurements.

In summary, PFM studies of $\mathrm{P}(\mathrm{VDF}-\mathrm{TrFE})$ thin films demonstrate nanoscale imaging and control of polarization in ultrathin ferroelectric polymer films. The imaging resolution was better than $5 \mathrm{~nm}$ and images showed single-domain regions corresponding to feature sizes averaging $25-50 \mathrm{~nm}$; the observed polarization patterns follow the irregular grain topography and the grains switch independently of each other. Arbitrary polarization patterns could be repeatedly written and erased with a resolution limited by the grain size, showing that these films are suitable for use in high-density data storage and retrieval at densities exceeding $250 \mathrm{Gbits} / \mathrm{in}^{2}$ and ferroelectric devices, ultimately down to the molecular level.

Work at the Oak Ridge National Laboratory (ORNL) was supported by LDRD funds (B.J.R., S.J., and S.V.K.). ORNL is operated by UT-Battelle for the U.S. Department of Energy under Contract No. DE-AC05-00OR22725. Work at the University of Nebraska (J.K., S.D., and V.M.F.) was supported by the National Science Foundation and the Nebraska Research Initiative. The authors are grateful to V. Meunier for insightful discussions.

${ }^{1}$ X. B. Zheng, P. T. Lai, B. Y. Liu, B. Li, and T. C. Cheng, Sens. Actuators, A 63, 147 (1997).

${ }^{2}$ T. D. Binnie, H. J. Weller, Z. He, and D. Setiadi, IEEE Trans. Ultrason. Ferroelectr. Freq. Control 47, 1413 (2000).

${ }^{3}$ H. Kohlstedt, Y. Mustafa, A. Gerber, A. Petrau, M. Fitsilis, R. Meyer, U. Böttger, and R. Waser, Microelectron. Eng. 80, 296 (2005).

${ }^{4}$ S. Ducharme, T. J. Reece, C. M. Othon, and R. K. Rannow, IEEE Trans. Device Mater. Reliab. 5, 720 (2005).

${ }^{5}$ R. G. Kepler and R. A. Anderson, Adv. Phys. 41, 1 (1992).

${ }^{6}$ A. J. Lovinger, Science 220, 1115 (1983).

${ }^{7}$ A. V. Bune, V. M. Fridkin, S. Ducharme, L. M. Blinov, S. P. Palto, A. V. Sorokin, S. G. Yudin, and A. Zlatkin, Nature (London) 391, 874 (1998).

${ }^{8}$ S. Ducharme, V. M. Fridkin, A. Bune, L. M. Blinov, S. P. Palto, and S. G. Yudin, Phys. Rev. Lett. 84, 175 (2000).

${ }^{9}$ P. Günther, J. Glatz-Reichenbach, and K. Dransfeld, J. Appl. Phys. 69, 7895 (1991).

${ }^{10}$ H. Birk, J. Glatz-Reichenbach, Li-Jie, E. Schreck, and K. Dransfeld, J. Vac. Sci. Technol. B 9, 1162 (1991).

${ }^{11}$ P. Günther and K. Dransfeld, Appl. Phys. Lett. 62, 1137 (1992).

${ }^{12}$ K. Matsushige, H. Yamada, H. Tanaka, T. Horiuchi, and X. Q. Chen, Nanotechnology 9, 208 (1998).

${ }^{13}$ K. Kimura, K. Kobayashi, H. Yamada, T. Horichi, K. Ishida, and K. Matsushige, Appl. Phys. Lett. 82, 4050 (2003).

${ }^{14}$ K. Noda, K. Ishida, A. Kubono, T. Horiuchi, H. Yamada, and K. Matsushige, Jpn. J. Appl. Phys., Part 1 40, 4361 (2001).

${ }^{15}$ K. Matsushige and H. Yamada, Ann. N.Y. Acad. Sci. 960, 1 (2002).

${ }^{16}$ C. Ludwig, G. Eberle, G. Gompf, J. Petersen, and W. Eisenmenger, Ann. Phys. (N.Y.) 2, 323 (1993).

${ }^{17} \mathrm{~K}$. Verkhovskaya, thesis, Russian Academy of Sciences, 1996.

${ }^{18}$ Y. Luo et al. (unpublished).

${ }^{19}$ L. M. Blinov, R. Barberi, S. P. Palto, M. P. De Santo, and S. G. Yudin, J. Appl. Phys. 89, 3960 (2001).

${ }^{20}$ A. Sorokin, S. Palto, L. Blinov, V. Fridkin, and S. Yudin, Mol. Mater. 6, 61 (1996).

${ }^{21}$ S. Ducharme, S. P. Palto, and V. M. Fridkin, in Ferroelectric and Dielectric Thin Films, edited by H. S. Nalwa (Academic, San Diego, CA, 2002), pp. 545-591.

${ }^{22}$ M. Bai, A. V. Sorokin, D. W. Thompson, M. Poulsen, S. Ducharme, C. M. Herzinger, S. Palto, V. M. Fridkin, S. G. Yudin, V. E. Savchenko, and L. K. Gribova, J. Appl. Phys. 95, 3372 (2004).

${ }^{23}$ S. Jesse, A. P. Baddorf, and S. V. Kalinin, Appl. Phys. Lett. 88, 062908 (2006).

${ }^{24}$ A. V. Sorokin, V. M. Fridkin, and S. Ducharme, J. Appl. Phys. 98, 044107 (2005). 